\title{
Design of an Ultra-Low Power SA-ADC with Medium/High Resolution and Speed
}

\author{
Andrea Agnes, Edoardo Bonizzoni, and Franco Maloberti \\ Department of Electronics \\ University of Pavia \\ Via Ferrata, 1, 27100 Pavia, ITALY \\ [andrea.agnes, edoardo.bonizzoni, franco.maloberti]@unipv.it
}

\begin{abstract}
Design strategies for power effective medium/high resolution Successive-Approximation ADC are discussed. The study considers reducing the power of the capacitive array with suitable capacitive attenuators that do not need using non-unity capacitors. The design of minimum power comparators is analyzed and a novel comparator scheme, named time-domain comparator, is described. The proposed methodologies, verified with a test design, is capable to provide 12-bit with $50-\mathrm{kHz}$ signal band and 1-V supply. The achieved FoM is $14 \mathrm{fJ} / \mathrm{conv}-$ level, which is well below the state-of-the-art.
\end{abstract}

\section{INTRODUCTION}

In energy-limited applications, such as wireless sensor networks and portable instruments, the use of ultra-low power ADCs extends the battery life and, possibly, allows autonomous operations. The successive approximation (SA) algorithm is a suitable low-power solution, [1 - 4], when the signal band is in up to about $1 \mathrm{MHz}$ and the resolution ranges between 10 and 12 bit. The SA architecture uses a comparator, a subtracting DAC together with its logic, and the successive approximation register (SAR) implementing the SA algorithm. The conversion starts with the sampling of the input signal and uses a clock period per bit. Therefore, the sampling rate is lower than the clock by a $(\mathrm{N}+1)$ factor. Since a capacitive array of equal unity capacitors achieves the subtraction DAC and the sampling function, the required power is related to the capacitances charge and discharge. With relatively low clock frequencies, the power consumption is in the $\mu \mathrm{W}$ range. A conventional scheme of voltage comparator consists in the cascade of a preamplifier and a latch. With submicron technologies, the capacitances to be charged and discharged are few ten of $\mathrm{fF}$. Therefore, even for the comparator, the required power is very low. Finally, the limited complexity of the SAR logic and the low clock frequency enable very low power consumption. As a result, the figure of merit (FoM) defined by

$$
F o M=\frac{P}{2^{E N O B} \cdot 2 \cdot f_{B}}
$$

where ENOB is the effective number of bits and $f_{B}$ is the bandwidth, can be as low as various tens of fJ/conv-level.

This paper discusses various design strategies to obtain a very low FoM, well below the state-of-the-art. The proposed methods and the use of a new comparator scheme are used in a test-design, obtaining a simulated FoM of $14 \mathrm{fJ} /$ conv-level almost verified on silicon, as synthetically described in [5].

\section{CAPACITIVE ARRAY}

The array of binary weighted capacitors of Fig. 1 realizes the sampling of the input signal and the charge redistribution by means of switches, controlled by the SAR logic, which connect the capacitors bottom plates to $V_{c m} \pm V_{R} / 2$. The power consumed by the capacitors affects the input terminal and the references. For the former contribution, notice that the SA algorithm gives rise to a redistribution of charges and leaves the array charged at the previous input sample at the end of the conversion. Therefore, for each sample, the input terminal must provide a charge to the total capacitance $C_{T}$ of the array given by

$$
Q\left(n T_{s}\right)=C_{T}\left[V_{i n}\left(n T_{s}\right)-V_{i n}\left(n T_{s}-T_{s}\right)\right]
$$

that increases with the input frequency and becomes maximum at Nyquist. Therefore, a full-scale sine wave at the Nyquist frequency drains a maximum current $I_{\text {in }}=C_{T} V_{R} / T_{s}$.

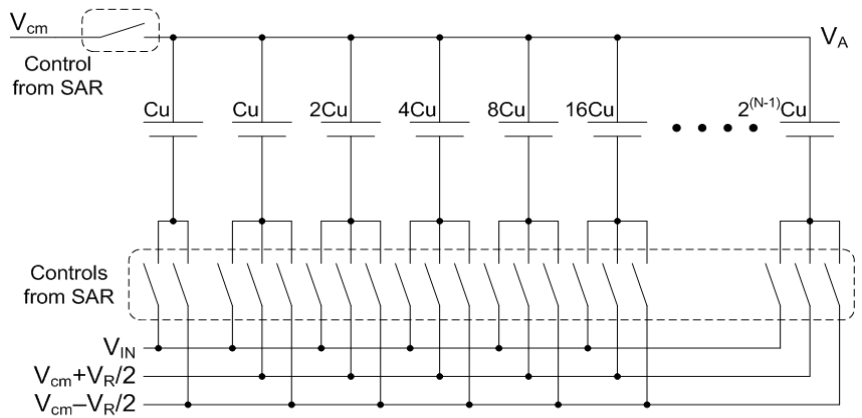

Figure 1. Binary weighted capacitors array. 


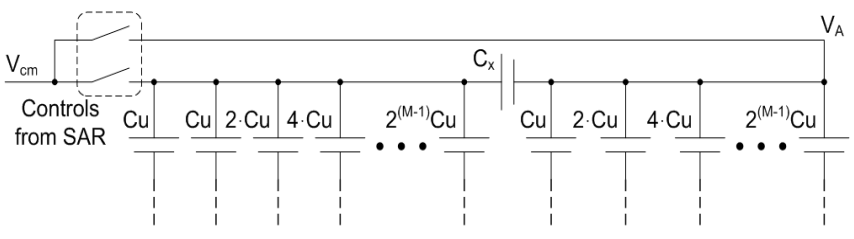

Figure 2. Binary capacitors arrays with attenuation capacitor.

The minimum total capacitance of the array is limited by the $2 k T / C$ noise associated to the array charging and discharging. Since the power of the quantization noise is $\mathrm{V}_{\mathrm{R}}{ }^{2} / 2^{2 \mathrm{~N}} / 12$, it is necessary to verify the condition

$$
\mathrm{C}_{\mathrm{T}}>\frac{24 \cdot \mathrm{kT} \cdot 2^{2 \cdot \mathrm{N}}}{\mathrm{V}_{\mathrm{R}}^{2}}
$$

For $V_{R}=1 \mathrm{~V}$ and $N=12$, the minimum value of $C_{T}$ is $1.64 \mathrm{pF}$ and the corresponding current $I_{i n}$ is equal to $0.164 \mu \mathrm{A}$. The figure is very low, but it is also necessary to account for the minimum unity capacitance that gives rise to a suitable mismatch error. As known, the capacitance accuracy is inversely proportional to the plate area, $A_{C}: \Delta C^{2} / C^{2}=K_{C} / A_{C}$. Moreover, it is necessary to account for the mismatch caused by the parasitic metal connections and switches. Modern technologies obtain very low values for $K_{C}$. However, overall limitations and a suitable margin suggest using $C_{U}>40 \mathrm{fF}$. Therefore, the use of a plain 12-bit binary array gives rise to $C_{T}=2{ }^{12} C_{U}>164 \mathrm{pF}$, a limit much larger that what established by the $2 k T / C$ limit. Accordingly, $I_{\text {in }}$ becomes about $16.4 \mu \mathrm{A}$.

The power drawn from the reference generator depends on the input amplitude and the resulting SA cycle. In average, the charge flowing from the reference generators to sustain the charge redistribution is about $1 / 2 C_{T} V_{R}$. Therefore, the average current is $I_{R}=1 / 2 C_{T} V_{R} / T_{s}$, half of the current drawn from the input terminal, considering a full scale sine wave at Nyquist.

The above study shows that if the minimum value of $C_{U}$ constrains $C_{T}$, an array of $2^{N}$ binary array consumes significant power, making convenient the use of an attenuation capacitance as shown in Fig. 2. Since the value of $C_{2}$ is scaled by a factor $k=2^{M}$, than the series of $C_{1}$ and $C_{x}$ must become $C_{1} / k$

$$
\frac{C_{1} C_{x}}{C_{1}+C_{x}}=\frac{C_{1}}{k}
$$

resulting in $C_{x}=C_{1} /(k-1)$. If $C_{1}=2^{m} C_{U}$, as normally chosen, $C_{x}$ is a fractional value that is difficult to realize (and this is the main limit to the use of the method). Instead, if $C_{1}=\left(2^{M}-1\right) C_{U}, C_{x}=C_{U}$ and this is positive. Moreover, having $C_{1}$ made by $\left(2^{m}-1\right)$ unity elements is not a big limit because there is a unity capacitance always connected to the lower reference. Its role is to have equivalent DAC full scale at 1 LSB below $V_{c m}+V_{R} / 2$. If that capacitor is removed, the full scale becomes $V_{c m}+V_{R} / 2$ with a negligible gain error $\alpha$. This result is an improvement of the technique proposed in [6].

It is also possible to extend the method to two attenuating capacitors, but having unity scaling capacitances is more problematic. The method described above with two scaled sections is not effective because the gain error $\alpha$ is inessential

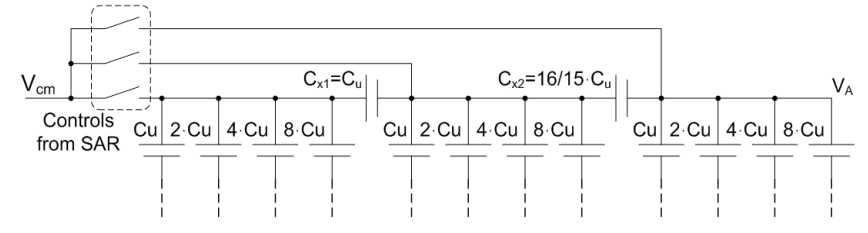

(a)

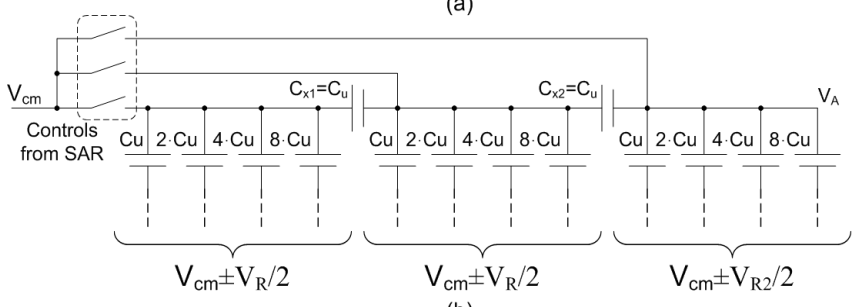

(b)

Figure 3. Binary capacitors arrays with two attenuation capacitors.
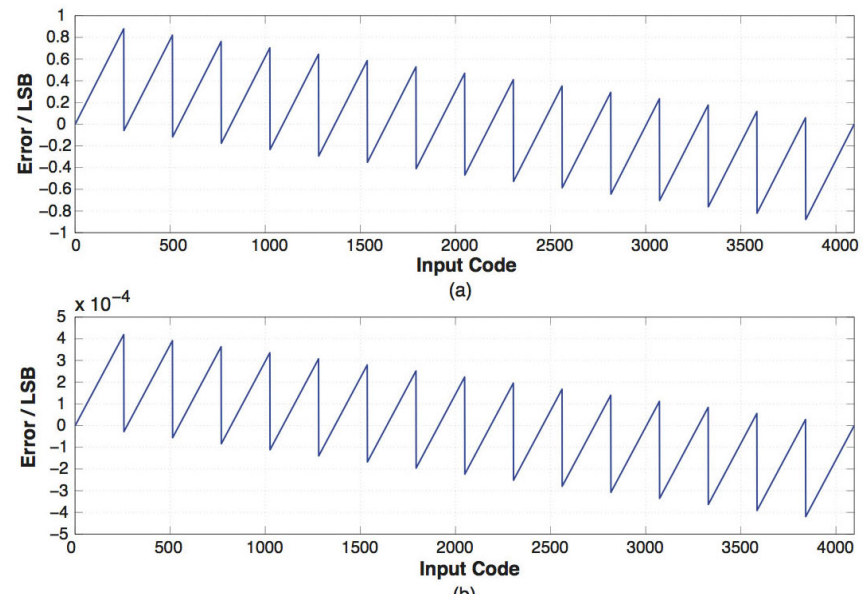

(b)

Figure 4. (a): INL of Fig. 3(a) scheme; (b): INL of Fig. 3(b) scheme.

when caused at the output, but it is responsible of a periodic 1-LSB INL if the architecture uses another attenuating section. Fig. 3(a) shows a $4+4+4$ binary arrays with one unity scaling capacitance, $C_{x 1}$, and the other, $C_{x 2}$, equal to $16 / 15 C_{U}$. Fig. 4 (a) shows the INL determined by this scheme. The INL worsens if even the second scaling element equals $C_{U}$. However, the gain error caused by the LSB scaling and the one produced by changing $C_{x 2}$ from $16 / 15 C_{U}$ to $C_{U}$ (Fig. 3 (b)) can be corrected by scaling the reference voltage in the MSB array. The use of $V_{c m} \pm V_{R 2} / 2$ with $V_{R 2}=1.0586 V_{R}$ allows obtaining the INL of Fig. 4 (b), that is lower than $\pm 0.0005 \mathrm{LSB}$. The MSB references, $V_{R 2}$, can be possibly obtained by an off-line calibration at the converter turn-on.

\section{COMPARATOR}

The second element that consumes power is the comparator. Since the input differential signal of a latch should be at least $20 \mathrm{mV}$ to avoid latching errors, it is necessary to use a preamplifier. The LSB of a 10-12-bit ADC is a fraction of $\mathrm{mV}$; therefore, the differential dynamic gain of the preamplifier should be in the $30-60 \mathrm{~dB}$ range. The transconductance of the input pair used in the preamplifier 
with input voltage $V_{\text {in }}$ determines a signal current, $g_{m} V_{i n}$, that charges a parasitic capacitance, $C_{p}$, for the preamplification time, $T_{p}$. The output voltage at the latch time becomes

$$
V_{\text {out }}=V_{\text {in }} \frac{g_{m} T_{p}}{C_{p}}=V_{\text {in }} \frac{I_{B} T_{p}}{m V_{T} C_{p}}
$$

where $I_{B}$ is the bias current of the input pair. Moreover, it is assumed that the MOS transistors of the input pair are in weak inversion $(m \approx 2.2)$. Equation (4) determines the minimum current for a given clock frequency, dynamic gain, $V_{L S B}$ and parasitic capacitance loading the preamplifier output. $C_{p}$ is given by the parasitic of the preamplifier output and the input parasitic of the latch. Assuming a preamplifier made by a cascode structure, $C_{p}$ for $0.13-0.18 \mu \mathrm{m}$ CMOS technology is estimated to be $40 \mathrm{fF}$. With $V_{L S B}=0.2 \mathrm{mV}, T_{p}=380 \mathrm{~ns}$, and $V_{\text {out }}=20 \mathrm{mV}$, the bias current $I_{B}$ equals $0.76 \mu \mathrm{A}$.

Even if this current consumption is already very low, the figure can be improved by using a new proposed time-domain comparator structure that, instead of operating in the voltage domain, transforms the input voltage into a pulse whose duration is compared with half period of the master clock. Fig. 5 shows the circuit schematic of the voltage-to-time ( $V 2 T)$ cell, used to generate the pulse. The input voltage $V_{A}$ establishes a current through $R_{D}$ that charges capacitor $C_{L}$.

When the signal $\Phi_{C}$ is low, transistors $M_{1}$ discharges the capacitor $C_{L}$. In the meantime, transistor $M_{4}$ discharges the parasitic capacitor $C_{P}$ to cancel out any memory of the previous conversion. When $\Phi_{C}$ rises, transistors $M_{2}$ turns on and the current generator made by $M_{3}$ and $R_{D}$ charge $C_{L}$ at constant rate. If the input voltage is constant and produces $V_{R}$ across $R_{D}$, then

$$
I_{D}=\frac{V_{R}}{R_{D}}
$$

When, after a given time $T_{d}$, the voltage across $C_{L}, V_{C}$, crosses the threshold of $M_{5}, \mathrm{Out}_{V 2 T}$ rises up, driven by the tapered inverter chain. $O u t_{V 2 T}$ drives also the logic which turns off $M_{2}$ for saving power, as the operation avoids to complete discharge $C_{L}$. Fig. 6 conceptually shows the V2T cell operation. For the used configuration $T_{d}$ increases when the input voltage is reduced and vice-versa. Moreover, since the parasitic capacitor $C_{P}$ is discharged by $M_{4}$, its series connection with $C_{L}$ gives an initial charging of $C_{L}$ itself. Therefore, voltage $V_{C}$ immediately falls to

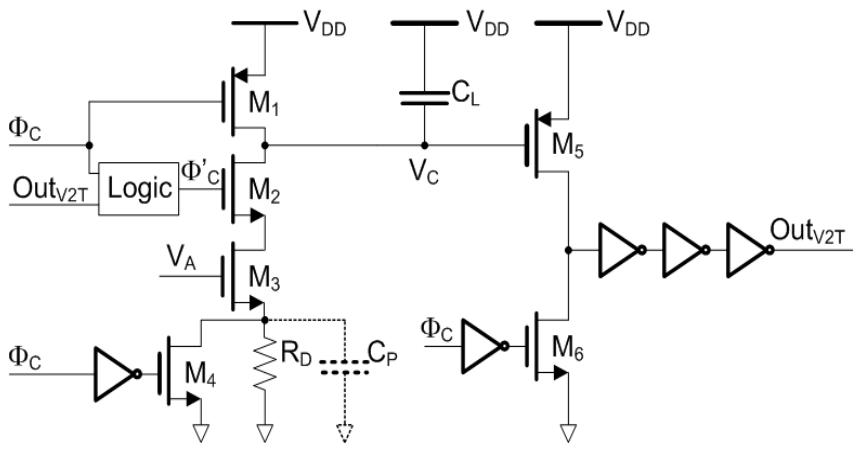

Figure 5. V2T cell schematic diagram.

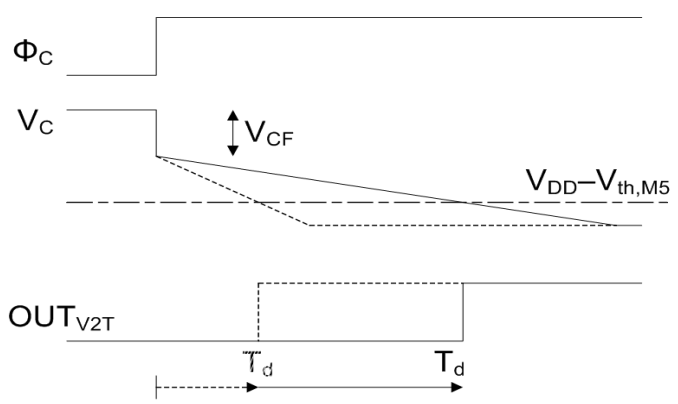

Figure 6. V2T main signal waveforms.

$$
V_{C F}=V_{D D} \frac{C_{P}}{C_{L}+C_{P}}
$$

Then, the constant rate discharge of $C_{L}$ starts. The initial drop of $V_{C}$ has positive effects because it reduces $T_{d}$ without increasing the discharge current. Splitting $C_{L}$ in two parts, $C_{L 1}$ and $C_{L 2}$, enhances the drop. $C_{L 1}$ is connected to $V_{D D}$ as in the scheme, $C_{L 2}$ is connected between $V_{D D}$ and the source of $M_{2}$ and pre-charged to $V_{D D}$ by a switch that ties the source of $M_{2}$ to ground during $\Phi_{C}$. Neglecting $C_{P}$, the drop of $V_{C}$ becomes $V_{D D}\left(C_{L 2} / C_{L 1}+C_{L 2}\right)$.

Fig. 7 shows the block diagram of the comparator together with its timing. It consists of a $V 2 T$ cell and a flip-flop delay $(F F D)$. The phase $\Phi_{C}$ used to pre-charge $C_{L}$ is on for a quarter of the clock. A delay of the $O u t_{V 2 T}$ pulse larger than $3 / 4 T_{C K}$ is revealed by the $F F D$ clocked by the master clock, $C K$. The time-domain comparator output voltage, $O u t_{c o m p}$, is then used as input of the $S A R$. Slightly after the crossing of the threshold, the logic opens $M_{2}$ and stops the flow of current through $R_{D}$. Therefore, if $\Delta V_{C}$ is the drop of the voltage $V_{C}$ giving rise to the bit detection, the energy per clock period consumed by $C_{L}$ is $C_{L} \cdot \Delta V_{C}^{2}$.

The design parameters of the $V 2 T$ are determined by the required accuracy that, by turn, is controlled by three main factors: the $k T / C$ noise, the input referred noise of $M_{5}, v_{n, 5}$, and the minimum distance between clock and $D$ input in the $F F D$.

Since the time for discharging $C_{L}$ by $\Delta V_{C}$ is $T_{0}=3 / 4 T_{C K}$, the bias value of $V_{A}$ must produce across $R_{L}$ a drop voltage $\Delta V_{R}$ such that $\Delta V_{C} / \Delta V_{R}=T_{0} / R_{L} C_{L}$. Therefore, an $L S B$ added to the bias of $V_{A}$ determine a $\delta V_{C}=L S B \cdot T_{0} / R_{L} C_{L}=$ $L S B \cdot \Delta V_{C} \Delta V_{R}$ variation at $t=T_{0}$.

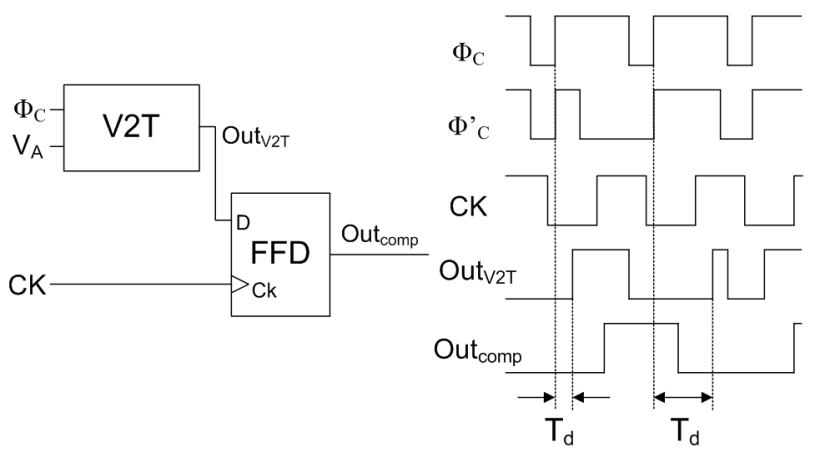

Figure 7. Time-domain block diagram. 
Therefore, the amplification of the LSB by $A_{0}=\Delta V_{C} / \Delta V_{R}$ must be lower than the equivalent noise across $C_{L}$; i.e. the quadratic superposition of $\operatorname{sqrt}\left(2 k T / C_{L}\right)$ with $v_{n, 5}$. If $C_{L}=0.8 \mathrm{pF}$, $v_{n, 5}=130 \mu \mathrm{V}$ and $A_{0}=2$, the LSB, that equals the noise, is as low as $83 \mu \mathrm{V}$.

The nominal slope of the discharge of $V_{C L}$ is $\Delta V_{C} / T_{0}$; accordingly, a time uncertainty $\delta t$ in the operation of the $F F D$ corresponds to an input referred noise $\delta V_{A}=\delta t \Delta V_{C} / T_{0} / A_{0}$. With $\Delta V_{C}=0.2 \mathrm{~V}, T_{0}=0.5 \mu \mathrm{s}$ and $\delta t=250 \mathrm{ps}, \delta V_{A}=43 \mu \mathrm{V}$. The value of $R_{L}$ is $312 \mathrm{k} \Omega$ and the average consumed power is $0.26 \mu \mathrm{W}$.

\section{DESIGN EXAMPLE}

The proposed design methods and the use of a timedomain comparator have been verified with transistor level simulations using a $0.18-\mu \mathrm{m}$ CMOS technology.

Two 31- $C_{U}$ elements and a $C_{U}$ attenuating capacitor make the capacitive array. The nominal value of $C_{U}$ is $100 \mathrm{fF}$. The switches used in the subtracting DAC are made by complementary devices, except the one connected to $V_{c m}$ that is an $n$-channel transistor with clock boost, [7].

The minimum supply voltage that achieves a good comparator response is as low as $0.7 \mathrm{~V}$, because the only limit to reduce the supply voltage is given by the drop across $R_{D}$ and the threshold of $M_{3}$. The nominal used supply voltage is $1 \mathrm{~V}, V_{c m}$ and $V_{R}$ are $0.5 \mathrm{~V}$ and $1 \mathrm{~V}$, respectively. Notice that the scheme allows using a $V_{R}$ higher than the rail-to-rail provided that the switches are only $n$-channel with clock boost, [6]. The values of discharging capacitances $C_{L 1}$ and $C_{L 2}$ are $0.6 \mathrm{pF}$ and $0.2 \mathrm{pF}$, respectively, thus achieving an initial drop of $250 \mathrm{mV}$.
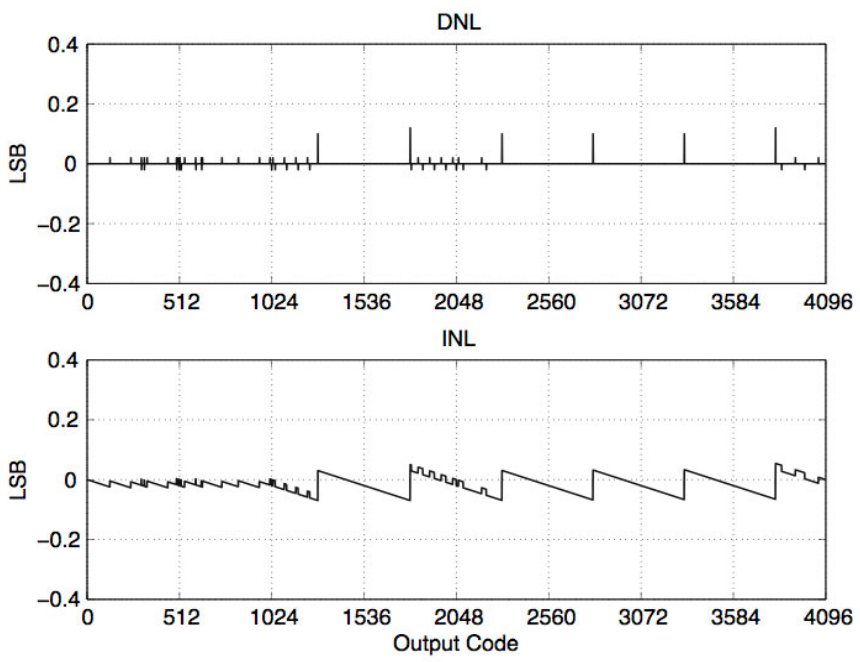

Figure 8. Simulated DNL and INL.

The simulations use a random error on unity capacitances with variance $\sigma=0.003$, value that is higher than what modern technologies obtain with $C_{U}=100 \mathrm{fF}$. Nevertheless, the obtained simulated results show typical DNL and INL plots like the one depicted in Fig. 8. It can be noted that both DNL and INL are less than \pm 0.2 LSB.
The spectra with $f_{C K}=1.3 \mathrm{MHz}, 12$-bit and a full-scale sinewave at $2.6 \mathrm{kHz}$ and $44.3 \mathrm{kHz}$ are shown in Fig. 9. The simulation results show SNRs of $70.8 \mathrm{~dB}$ and $68.6 \mathrm{~dB}$ respectively (11.8 and 11.1 ENOB) with a third harmonic below $90 \mathrm{~dB}$.

The total simulated power consumption is $3.1 \mu \mathrm{W}$ that allows obtaining a 14-fJ/conv-step of FoM. A similar circuit with a less sophisticated architecture of the time-domain comparator has been experimentally evaluated. The obtained ENOB is 9.4-bit which demonstrates the effectiveness of the approach providing a FoM of $56 \mathrm{fJ} /$ conv-level, [5].
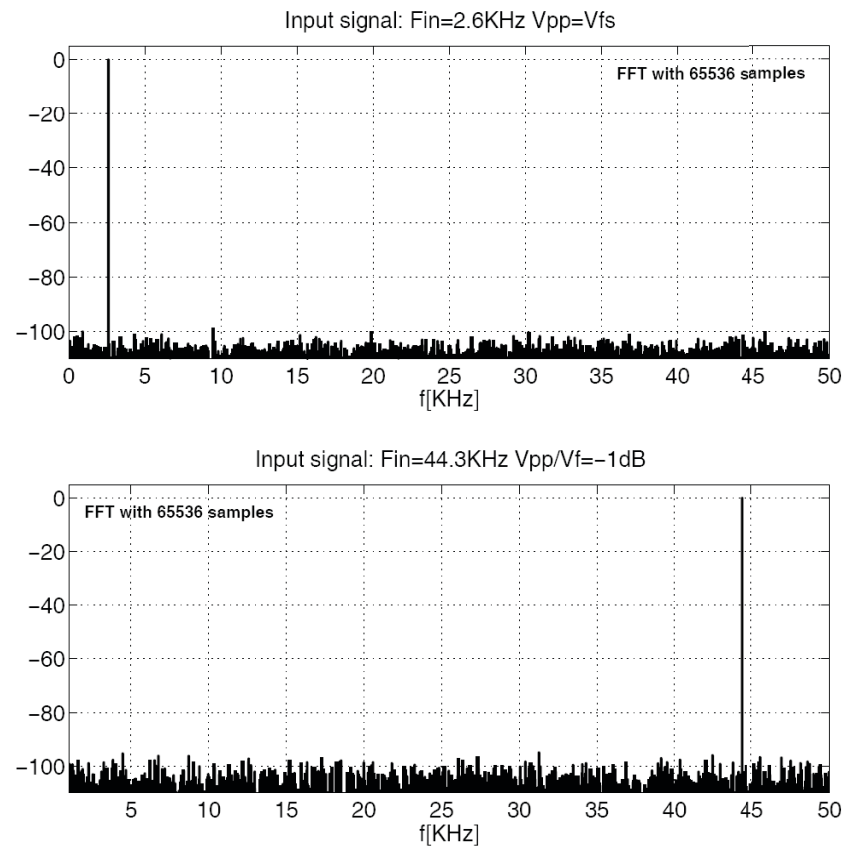

Figure 9. Output Spectrums with input sine wave @ $2.6 \mathrm{kHz}$ and $44.3 \mathrm{kHz}$.

\section{REFERENCES}

[1] F. Maloberti, "Data converters", Springer, 2007.

[2] M.D. Scott, B.E. Boser, and K.S.J. Pister, "An Ultralow-Energy ADC for Smart Dust" IEEE Journal of Solid State Circuits, Vol. 38, pp. 1123-1129, 2003.

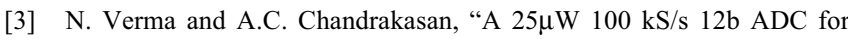
wireless application", IEEE International Solid-State Circuits Conference Dig. Tech. Papers (ISSCC), pp. 222-223, Feb. 2006.

[4] J.Sauerbrey, D. Schimitt-Landsiedel, R. Thewes, "A $0.5 \mathrm{~V}, 1 \mu \mathrm{W}$ Successive Approximation ADC", IEEE European Solid State Circuits Conference (ESSCIRC), pp. 247-250, 2002.

[5] A. Agnes, E. Bonizzoni, P. Malcovati, and F. Maloberti, "A 9.4 ENoB, $1 \mathrm{~V}, 3.8 \mu \mathrm{W}, 100 \mathrm{kS} / \mathrm{s}$ SAR-ADC with Time-Domain Comparator", to appear in 2008 IEEE International Solid-State Circuits Conference Dig. Tech. Papers (ISSCC).

[6] P. Confalonieri, M. Zamprogno, F. Girardi, G. Nicollini, and A. Nagari, "A $2.7 \mathrm{~mW} 1 \mathrm{MSps} 10 \mathrm{~b}$ Analog-to-Digital Converter with Built-in Reference Buffer and 1LSB Accuracy Programmable Input ranges", Proc. of IEEE European Solid State Circuits Conference (ESSCIRC), pp. 255-258, Sept.2004.

[7] D. Aksin, M. Al-Shyoukh, and F. Maloberti, "Switch Bootstrapping for Precise Sampling Beyond Supply Voltage", IEEE Journal of Solid State Circuits, pp. 1938-1943, Aug.2006. 